\title{
The Dynamics of Macrophage Infiltration into the Arterial Wall during Atherosclerotic Lesion Development in Low-Density Lipoprotein Receptor Knockout Mice
}

\author{
Dan Ye, ${ }^{*}$ Ying Zhao, ${ }^{*}$ Reeni B. Hildebrand, ${ }^{*}$ \\ Roshni R. Singaraja, ${ }^{\dagger}$ Michael R. Hayden, ${ }^{\dagger}$ \\ Theo J.C. Van Berkel, ${ }^{*}$ and Miranda Van Eck* \\ From the Division of Biopharmaceutics, * Leiden/Amsterdam \\ Center for Drug Research, Leiden University, Leiden, the \\ Netherlands; and the Centre for Molecular Medicine and \\ Therapeutics, ${ }^{\dagger}$ Children's and Women's Hospital, University of \\ British Columbia, Vancouver, British Columbia, Canada
}

Atherosclerosis is a progressive disease in which macrophages play an essential role. Macrophage infiltration into the arterial wall induces the development of an early atherosclerotic lesion. However, the dynamics of macrophage infiltration into the arterial wall during lesion progression remain poorly understood. In this study, low-density lipoprotein receptor knockout mice were fed a Western-type diet for 3, 6, 9, and 12 weeks to induce the formation of atherosclerotic lesions with different degrees of complexity. Subsequently, these mice underwent transplantation with bone marrow-overexpressing enhanced green fluorescent protein to track donor-derived cells, including macrophages. After 8 weeks of Western-type diet feeding after transplantation, macrophage infiltration was evaluated by immunohistochemical staining of donor-derived macrophages (enhanced green fluorescent protein-positive $\mathrm{F} 4 / 8^{+}$) in the aortic roots. We found that the growth of pre-existing initial lesions was mainly caused by continued recruitment of donor-derived macrophages into the arterial wall. Interestingly, macrophage infiltration into pre-existing more advanced lesions was largely impaired, likely because of the formation of fibrous caps. In addition, interference with the expression of macrophage ATP-binding cassette transporter 1, an ATP-binding cassette transporter involved in cellular cholesterol efflux and macrophage recruitment into tissues, affects the infiltration of macrophages into pre-existing early lesions but not into ad- vanced lesions. In conclusion, our data suggest that the dynamics of macrophage infiltration into the arterial wall vary greatly during atherogenesis and, thus, may affect the efficiency of pharmaceutical interventions aimed at targeting macrophage infiltration into the arterial wall. (Am J Pathol 2011, 178:413-422; DOI: 10.1016/j.ajpath.2010.11.007)

Atherosclerosis is a progressive disease characterized by the accumulation of lipid-laden macrophages and fibrous elements within the large arteries. Recent studies have suggested that atherosclerosis is a dynamic vascular disease, displaying both progression and regression of atherosclerotic lesions and marked changes in composition that affect plaque stability. ${ }^{1}$ Hypercholesterolemia, especially that resulting from high levels of very-low-density lipoprotein (VLDL) and low-density lipoprotein (LDL) cholesterol, is one of the key risk factors in the development of atherosclerotic lesions. ${ }^{2}$ However, hypercholesterolemic mice become resistant to atherosclerosis when bred to a macrophage-deficient background, ${ }^{3}$ illustrating the crucial role of macrophages in promoting lesion initiation and progression. Macrophages are transformed into foam cells on accumulation of (modified) lipoproteins, resulting in the formation of fatty streaks that represent the earliest detectable atherosclerotic lesions. Macrophages also play important roles in

Supported by the Netherlands Heart Foundation grants 2007T056 (D.Y.) and 2001T4101 (Y.Z.); by the Netherlands Organization for Scientific Research VIDI grant 917.66.301 (M.V.E.); and by the Canadian Institutes of Health Research. M.V.E. is an Established Investigator of the Netherlands Heart Foundation (grant 2007T056). M.R.H. is a University Killam Professor and holds a Canada Research Chair in Human Genetics and Molecular Medicine

Accepted for publication September 2, 2010.

Supplemental materials to this article can be found at http://ajp. amjpath.org or at doi:10.1016/j.ajpath.2010.11.007.

Address reprint requests to Dan Ye, Ph.D., Division of Biopharmaceutics, Gorlaeus Laboratory, Einsteinweg 55, 2333 CC Leiden, the Netherlands. E-mail: y.dan@lacdr.leidenuniv.nl. 
innate and acquired immune responses. ${ }^{4}$ They mediate inflammatory response by secreting various cytokines, chemokines, and growth factors, thus encouraging the recruitment of other cell types (eg, monocytes, T cells, fibroblasts, and smooth muscle cells) that promote atherogenesis. Moreover, macrophages produce a variety of matrixdegrading proteases that can affect plaque stability by inducing weakening of the fibrous cap., ${ }^{5,6}$ Thus, macrophages play essential roles in all stages of atherosclerotic lesion development. Although the critical role of macrophage infiltration in the initiation of atherosclerosis is generally accepted, the dynamics of macrophage infiltration into the arterial wall during atherosclerosis progression remain an open question.

The accumulation of macrophages in atherosclerotic lesions primarily depends on the infiltration of bone marrow-derived monocytes into the arterial wall. In this study, to clarify the dynamics of macrophage infiltration into the arterial wall during atherogenesis, LDL receptor knockout (KO) $\left(\mathrm{LDLr}^{-1-}\right)$ mice with pre-existing initial or more advanced atherosclerotic lesions underwent transplantation with bone marrow overexpressing enhanced green fluorescent protein (EGFP) to track donor-derived cells, including macrophages. We show that the ability of donor-derived macrophages to infiltrate into the arterial wall is influenced by the severity of the pre-existing lesions. This may have important implications for the design of pharmaceutical interventions aimed at targeting macrophage infiltration into the arterial wall. ATP-binding cassette transporter 1 (ABCA1)-dependent cholesterol efflux is a crucial factor in the prevention of excessive cholesterol accumulation in macrophages of the arterial wall and in their transformation into foam cells. ${ }^{7-10}$ Therefore, the concept that promotion of macrophage cholesterol efflux by up-regulating ABCA1 might prevent progression or even induce regression of atherosclerosis is remarkably attractive. Moreover, ABCA1 has also been implicated as a factor that is important for the recruitment of macrophages into tissues. ${ }^{9}$ For the design of new therapeutic strategies aimed at up-regulating $A B C A 1$, it is essential to quantify the effect of ABCA1 on macrophage infiltration into lesions. Therefore, studies were performed in which $\mathrm{LDLr}^{-1-}$ mice with pre-existing initial or advanced lesions underwent transplantation with $\mathrm{ABCA} 1 \mathrm{KO}$ or $\mathrm{ABCA} 1$-overexpressing bone marrow, also coexpressing EGFP. We found that overexpression of ABCA1 decreases macrophage recruitment specifically into early lesions, resulting in reduced lesion sizes. Our data suggest that preventing macrophage recruitment into early lesions provides a therapeutic strategy to reduce atherosclerosis burden. Increasing macrophage $A B C A 1$ may be one avenue of doing so.

\section{Materials and Methods}

\section{Mice}

C57BL/6 mice that express a transgene coding for EGFP under control of the human ubiquitin $\mathrm{C}$ promoter were obtained from the Jackson Laboratory (Bar Harbor, ME).
These mice, called UBC-EGFP/BL6, express GFP in all tissues examined, with high levels of GFP expression observed in hematopoietic cells (henceforth called EGFP/wild type [WT]). ${ }^{11}$ Mice homozygous for the null mutant $A B C A 1$ gene and expressing EGFP (henceforth called EGFP/ABCA1 KO) and mice overexpressing human ABCA1 bacterial artificial chromosome and EGFP (henceforth called EGFP/hABCA1) were generated by crossbreeding. All of the transgenic mice are on a C57BL/6J background. Homozygous $\mathrm{LDLr}^{-1-}$ mice (C57BL/6 background) were purchased from the Jackson Laboratory as mating pairs and bred at the Gorlaeus Laboratory (Leiden, the Netherlands). Mice received a regular chow diet $(4.3 \% \mathrm{wt} / \mathrm{wt}$ fat and no added cholesterol; RM3; Special Diet Services, Witham, UK) until the beginning of the study (mice aged 8 to 10 weeks), when $\mathrm{LDLr}^{-1-}$ mice were fed a Western-type diet (WTD) (15\% wt/wt cacao butter and 0.25\% wt/wt cholesterol; Diet W; Ab diets, Woerden, the Netherlands) to induce hypercholesterolemia and atherosclerosis. Animal experiments were performed at Gorlaeus Laboratory in accordance with national laws. All experimental protocols were approved by the Ethics Committee for Animal Experiments of Leiden University, Leiden, the Netherlands.

\section{Generation of Chimeras by Bone Marrow Transplantation}

To induce bone marrow aplasia, female $\mathrm{LDLr}^{-1-}$ mice without or with pre-existing atherosclerotic lesions were exposed to a single dose of 9 Gy (0.19 Gy/min, 200 kV, 4 $\mathrm{mA}$ ), using a Roentgen source (Andrex Smart 225; YXLON International, Copenhagen, Denmark) with a 6-mm aluminum filter 1 day before bone marrow transplantation (BMT). Bone marrow was harvested by flushing the femurs and tibias from the donor mice with PBS. Single-cell suspensions were prepared by passing the cells through a $30-\mu \mathrm{m}$ nylon gauze. Irradiated recipients received $0.5 \times 10^{7}$ bone marrow cells by intravenous injection into the tail vein. Drinking water was supplied with antibiotics (83-mg/L ciprofloxacin and 67-mg/L polymyxin B sulfate) and 6.5-g/L sucrose.

\section{Flow Cytometry (FACS)}

To determine the extent of repopulation of circulating blood cells with donor cells after transplantation, whole blood was collected each week after BMT. Blood cell suspension in PBS was detected for EGFP fluorescence by FACS analysis.

Eight weeks after BMT, mice that underwent transplantation were euthanized and the spleen and intestinal lymph nodes were harvested. Single-cell suspensions were prepared by passing the spleen and lymph nodes through a 30- $\mu \mathrm{m}$ nylon gauze. Leukocytes from the spleen were isolated by density-gradient centrifugation (Lympholyte; Cedarlane Laboratories, Hornby, Ontario, Canada) according to the manufacturer's protocol. Cell suspensions from the spleen and lymph nodes were incubated with $1 \%$ normal mouse serum in PBS and 
stained for surface markers $(0.5-\mu \mathrm{g}$ antibody/300,000 cells). All antibodies were purchased from eBiosciences, Halle, Belgium. Samples were analyzed by FACS. All data were acquired on an FACSCalibur and analyzed with software (CELLQuest; BD Biosciences, Heidelberg, Germany).

\section{Serum Lipid Analyses}

After an overnight fast, approximately $100 \mu \mathrm{L}$ of blood was drawn from each mouse by tail bleeding. Serum levels of total cholesterol (TC), free cholesterol (FC), and esterified cholesterol (CE) were determined using enzymatic colorimetric assays (Roche Diagnostics, Mannheim, Germany), with $0.03-\mathrm{U} / \mathrm{ml}$ cholesterol oxidase (Sigma Chemical Co, St. Louis, MO), 0.065-U/ml peroxidase, and $15-\mu \mathrm{g} / \mathrm{ml}$ cholesteryl esterase (Seikagaku, Tokyo, Japan) in reaction buffer (1.0 potassium phosphate buffer [KPi] $[\mathrm{pH}, 7.7]$ containing $0.01-\mathrm{mol} / \mathrm{L}$ phenol, 1-mmol/L 4-aminoantipyrine, 1\% polyoxyethylene-9-laurylether, and $7.5 \%$ methanol). The concentrations of phospholipids (PLs) and triglycerides (TGs) in serum were determined using enzymatic colorimetric assays (Spinreact S.A., Gerona, Spain and Roche Diagnostics, Mannheim, Germany respectively). Precipath I (Roche Diagnostics) was used as an internal standard. The absorbance was read at $490 \mathrm{~nm}$. The distribution of lipids over the different lipoproteins was determined by fractionation of $30 \mu \mathrm{L}$ of serum from each mouse using a 3.2 $\times$ 300-mm column (Superose 6, Smart-system; Pharmacia, Uppsala, Sweden). The TC, TG, and PL contents in the effluent were determined as previously described.

\section{Histological and Immunocytochemical Analysis of the Aortic Root}

To analyze atherosclerosis development, mice that underwent transplantation were euthanized; and hearts and aortas were perfused in situ with PBS for 20 to 30 minutes via a cannula in the left ventricle and subsequently stored in 3.7\% neutral-buffered formalin (Formal-Fixx; Shandon Scientific Ltd, Runcorn, UK). Cryostat sections of the aortic root $(10 \mu \mathrm{m})$ were collected and stained with oil red O/hematoxylin (Sigma Diagnostics, St Louis, MO). The mean lesion area (in $\mu \mathrm{m}^{2}$ ) was calculated from 10 consecutive sections, starting at the appearance of the tricuspid valves. Moreover, sections on separate slides were stained with a primary monoclonal antibody to monocyte/macrophage marker (MOMA-2) (rat antimouse IgG2b; dilution, 1:50; Research Diagnostics, Flanders, NJ) and a secondary antibody conjugated to alkaline phosphates (goat anti-rat IgG-alkaline phosphates; dilution, 1:200; Sigma, St. Louis, MO). MOMA-2 is a useful marker for broad detection of monocytes and macrophages. 5-bromo-4-chloro-3-indolyl-phosphate and nitroblue tetrazolim (Sigma) were used as enzyme substrates. In addition, sections were immunolabeled with a primary monoclonal antibody to F4/80 (rat antimouse; dilution, 1:200; BMA Biomedicals, Augst, Switzerland) for the detection of mature macrophages and a peroxidase-conjugated secondary antibody (Jackson ImmunoResearch Laboratories, Suffolk, UK). Masson trichrome staining (Sigma Diagnostics) was used to visualize collagen (blue staining). The macrophage and collagen contents of lesions were subsequently calculated as the percentage of mean positive area versus mean total lesion area using five consecutive sections per mouse by computer-aided morphometric analysis. A histological classification of atherosclerotic lesions in the aortic root was performed on oil red O/hematoxylin- and Masson's trichrome-stained sections according to the recommendations of the American Heart Association. ${ }^{12}$ Images were obtained with an image analysis system (Leica) consisting of a microscope (Leica DMRE) coupled to a camera and software (Leica Qwin Imaging: Leica Ltd, Cambridge, UK).

To track donor-derived macrophages, cryostat sections of the aortic root were double immunolabeled with the primary monoclonal antibodies: rat anti-mouse F4/80 for the detection of macrophages and goat anti-mouse JL-8 (dilution, 1:2,000; CLONTECH Laboratories, Inc, Palo Alto, CA) for the detection of EGFP. Secondary antibodies were conjugated to cyanine dye and fluorescein isothiocyanate (FITC) (Jackson ImmunoResearch Laboratories), respectively. Nuclei were stained with 4,6-diamidino-2-phenylindole (Serva Feinbiochemica, Heidelberg, Germany). Photomicrographs were obtained using a confocal laser scanning system (Bio-Rad Radiance 2100 MP) equipped with an inverted fluorescence microscope (Eclipse TE2000-U; Nikon, Melville, NY). The acquisition of images and the analysis of lesions were performed in a blinded fashion.

\section{Hepatic Lipid Composition and Liver Histological/Immunohistological Features}

Hepatic lipids were extracted according to the method of Bligh and Dyer. ${ }^{13}$ After dissolving the lipids in $2 \%$ Triton $\mathrm{X}-100$, the contents of FC, CE, PL, and TG in liver tissue were determined as previously described and expressed as micrograms per milligram of protein.

Cryostat sections of the liver $(8 \mu \mathrm{m})$ were collected and routinely stained with oil red O/hematoxylin (Sigma Diagnostics) for lipid visualization. In addition, to investigate the replacement of Kupffer cells after transplantation, liver sections were also double immunolabeled to detect macrophages and EGFP as previously described.

\section{Statistical Analyses}

Data were presented as mean \pm SEM. Statistical analyses were performed using one- and two-way analysis of variance with software (Graphpad Prism Software; Graphpad Software, Inc., San Diego, CA [http://www. graphpad.com]). The level of statistical significance was set at $P<0.05$. 
Table 1. Effect of WTD Feeding on Serum and Hepatic Lipid Levels in LDLr $^{-/}{ }^{-}$Mice*

\begin{tabular}{|c|c|c|c|c|c|}
\hline Group & Time receiving a WTD, wk & $\mathrm{FC}^{\dagger}$ & $\mathrm{CE}^{\dagger}$ & $\mathrm{PL}^{\dagger}$ & $\mathrm{TG}^{\dagger}$ \\
\hline \multicolumn{6}{|c|}{ Serum data } \\
\hline 1 & 0 & $78 \pm 4$ & $338 \pm 18$ & $497 \pm 18$ & $143 \pm 13$ \\
\hline 2 & 3 & $221 \pm 14^{\ddagger}$ & $732 \pm 11^{\S}$ & $687 \pm 13^{\ddagger}$ & $154 \pm 19$ \\
\hline 3 & 6 & $362 \pm 25^{\pi}$ & $1712 \pm 74^{\pi}$ & $985 \pm 45^{\S}$ & $164 \pm 38$ \\
\hline 4 & 9 & $216 \pm 36^{\ddagger}$ & $1643 \pm 58 \pi$ & $1023 \pm 64^{\S}$ & $147 \pm 29$ \\
\hline 5 & \multicolumn{4}{|c|}{ Hepatic data } & $124 \pm 23$ \\
\hline 1 & 0 & $16 \pm 1$ & $8 \pm 2$ & $70 \pm 4$ & $156 \pm 6$ \\
\hline 2 & 3 & $21 \pm 2^{\ddagger}$ & $32 \pm 5^{\S}$ & $74 \pm 3$ & $134 \pm 9$ \\
\hline 3 & 6 & $28 \pm 2^{\S}$ & $47 \pm 3^{\S}$ & $77 \pm 4$ & $130 \pm 7$ \\
\hline 4 & 9 & $36 \pm 2^{1 \|}$ & $60 \pm 7^{\uparrow \|}$ & $83 \pm 8^{\ddagger}$ & $114 \pm 5$ \\
\hline 5 & 12 & $39 \pm 1^{9 \|}$ & $68 \pm 3^{9 \| 1}$ & $81 \pm 6$ & $113 \pm 7$ \\
\hline
\end{tabular}

WTD, Western-type diet; LDLr, low-density lipoprotein receptor; FC, free cholesterol; CE, cholesterol ester; PL, phospholipid; TG, triglyceride.

${ }^{*}$ For all groups, $\mathrm{n}=6$. For serum data, values are given in $\mathrm{mg} / \mathrm{dL}$; and for hepatic data, values are given in $\mu \mathrm{g} / \mathrm{mg}$. Serum levels of $\mathrm{FC}, \mathrm{CE}, \mathrm{PL}$, and TG were determined in $\mathrm{LDLr}^{-1-}$ mice fed a regular chow diet (baseline, group 1) or a high-cholesterol WTD for 3, 6, 9 , and 12 weeks (groups 2 to 5) Moreover, hepatic lipids were extracted from these WTD-fed LDLr ${ }^{-1}$ mice; and hepatic levels of FC, CE, PL, and TG were determined.

${ }^{\dagger}$ Data are given as mean \pm SEM.

${ }_{\ddagger}^{\ddagger} P<0.05$ versus $\mathrm{LDLr}^{-1-}$ mice receiving chow (group 1)

$\$ P<0.01$ versus $\mathrm{LDLr}^{-1-}$ mice receiving chow (group 1).

$\pi P<0.001$ versus $\mathrm{LDLr}^{-1-}$ mice receiving chow (group 1)

$\| P<0.05$ versus $\mathrm{LDLr}^{-1-}$ mice receiving a WTD for 6 weeks (group 3).

\section{Results}

\section{Generation of LDLr ${ }^{-1-}$ Mice with Pre-existing Atherosclerotic Lesions by WTD Feeding}

To induce the development of atherosclerotic lesions with different degrees of complexity, $\mathrm{LDLr}^{-1-}$ mice received a regular chow diet or were fed a WTD for 3, 6, 9, and 12 weeks. On the chow diet, most serum cholesterol in $\mathrm{LDLr}^{-1-}$ mice is transported by LDL and high-density lipoprotein (data not shown). Serum TC was increased by approximately twofold $(P<0.01)$ and approximately fivefold $(P<0.001)$ after 3 and 6 weeks of WTD feeding, respectively; and the increases in TC were primarily in the
VLDL/LDL cholesterol fractions (data not shown). As shown in Table 1, serum levels of FC and CE were induced by 3-and 6-week WTD feeding. As compared to 6 weeks, 9 and 12 weeks of WTD feeding led to less profound increases in serum FC levels. In addition, serum levels of $C E$ and $P L$, but not $T G$, were also induced by WTD feeding (Table 1). The elevated PL levels were primarily because of increases in the VLDL/LDL fractions (data not shown).

The liver of WTD-fed LDLr ${ }^{-1-}$ mice displayed massive accumulation of lipids, as examined by oil red $\mathrm{O}$ staining (data not shown) and hepatic lipid composition analysis (Table 1). The $\mathrm{LDLr}^{-/-}$mice that were challenged with a
A

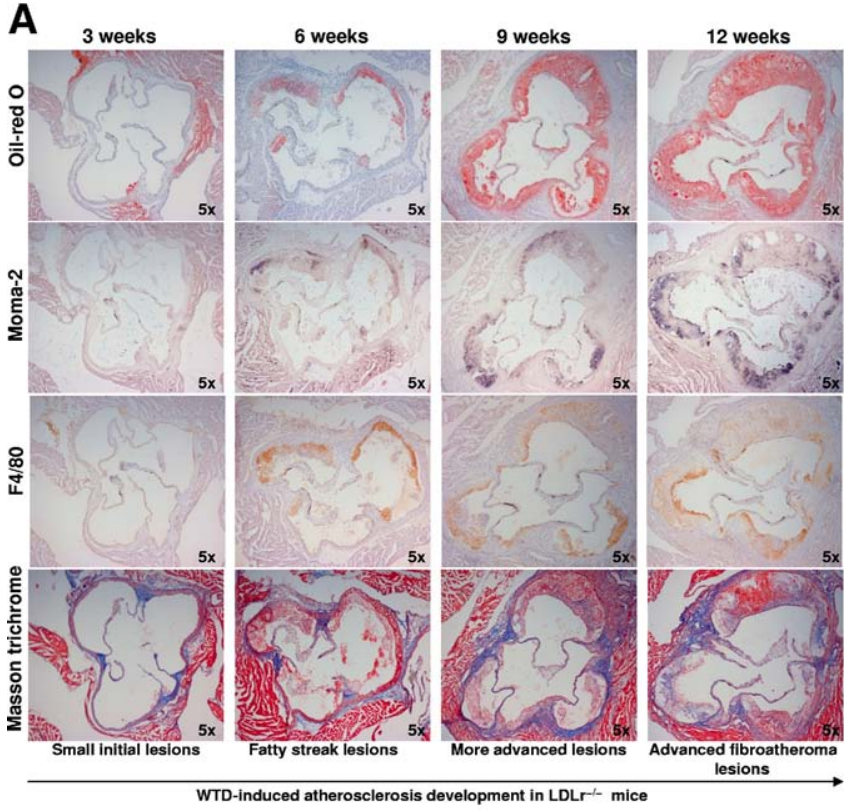

B
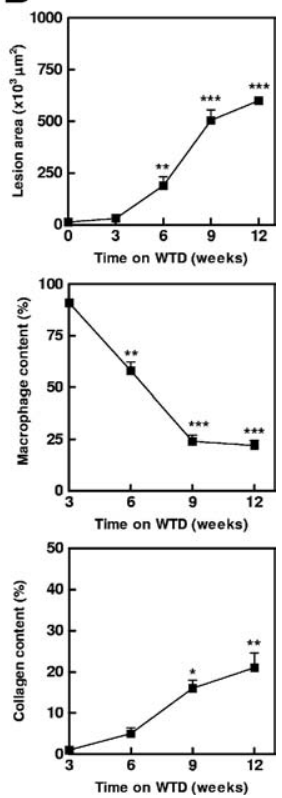

Figure 1. A Western-type diet (WTD) induces atherosclerosis development in low-density lipoprotein receptor (LDLr) ${ }^{-/-}$mice. A: Atherosclerotic lesion development was analyzed in the aortic root of $\mathrm{LDLr}^{-1-}$ mice after $0,3,6,9$, and 12 weeks of WTD feeding. Representative images for oil red O, MOMA-2 (monocytes and macrophages), F4/80 (macrophages), and Masson trichrome (collagen) staining (original magnification, $\times 50$ ). B: The mean lesion area was calculated from oil red $\mathrm{O} /$ hematoxylin-stained cross sections of the aortic root at the level of the tricuspid valves. The macrophage and collagen contents of the lesions were quantified in five consecutive sections. Values represent the mean of six mice per group. ${ }^{*} P<0.05,{ }^{*} P<0.01$, and ***: $P<0.001$ versus a chow diet 
WTD for a longer period (ie, 9 and 12 weeks) displayed higher levels of intrahepatic cholesterol, mainly FC and CE.

Next, atherosclerotic lesion development was analyzed in the aortic root of LDLr ${ }^{-1-}$ mice at different points after the initiation of the WTD challenge. No atherosclerotic lesions were found in LDLr ${ }^{-1-}$ mice fed a chow diet. After 3-week WTD feeding, small initial lesions $\left(31 \pm 7 \times 10^{3} \mu \mathrm{m}^{2}, n=6\right)$ were formed, primarily composed of macrophage-derived foam cells. The lesion size increased further after 6,9 , and 12 weeks of WTD feeding $\left(190 \pm 43 \times 10^{3} \mu \mathrm{m}^{2}, n=6 ; 505\right.$ $\pm 51 \times 10^{3} \mu \mathrm{m}^{2}, n=6$; and $600 \pm 11 \times 10^{3} \mu \mathrm{m}^{2}, n=6$, respectively) (Figure 1, A and $\mathrm{B}$ ). Based on lesion composition, plaques were classified as fatty streak lesions (58\% $\pm 4.2 \%$ for macrophage content and $5 \% \pm 1.4 \%$ for collagen content), more advanced lesions (24\% $\pm 3.1 \%$ for macrophage content and $16 \% \pm 2.0 \%$ for collagen content), and advanced fibroatheroma lesions (22\% $\pm 2.5 \%$ for macrophage content and $21 \% \pm 3.5 \%$ for collagen content) after 6,9 , and 12 weeks of WTD feeding, respectively (Figure 1B).

\section{Generation of $L D L r^{-/-}$Mice with Pre-Existing Atherosclerotic Lesions and Overexpressing EGFP in Macrophages}

To assess the dynamics of macrophage infiltration into the arterial wall during atherosclerosis development, we performed a BMT in which bone marrow from EGFP/WT mice was transplanted into $\mathrm{LDLr}^{-1-}$ mice that had been fed a WTD for $0,3,6,9$, and 12 weeks before BMT $\left(\right.$ EGFP/WT $\rightarrow \mathrm{LDLr}^{-/-}$). The animals that underwent transplantation were sacrificed after $8,11,14,17$, and 20 weeks of WTD feeding (ie, 8 weeks of WTD feeding after BMT) (Figure 2A). To exclude potential direct effects of irradiation on plaque morphological features, we compared initial lesions with advanced lesions in LDLr KO mice before irradiation and 3 days after irradiation. Neither lesion size nor lesion composition was significantly changed as a result of irradiation (data not shown).

Regardless of the duration of WTD feeding before $\mathrm{BMT}$, circulating whole blood cells were quantitatively replaced by donor-derived cells $\left(\mathrm{EGFP}^{+}\right)$, starting at 6 days after BMT and reaching a level of $99 \% \pm 0.2 \%$ at 8 weeks after transplantation (see Supplemental Figure S1 at http://ajp.amjpathol.org). This indicated that the bone marrow transfers were successful.

Independently of the duration of WTD feeding before transplantation, BMT resulted in a temporary decrease in serum TC levels (approximately $22 \%$ to $35 \%$ ), primarily because of significantly reduced VLDL/LDL cholesterol levels and moderately decreased high-density lipoprotein cholesterol levels (data not shown). This effect is specific for mice on a WTD because it was not observed in mice that had undergone transplantation while being fed a regular chow diet. $^{9,10}$ Nevertheless, the new steady-state serum choles-

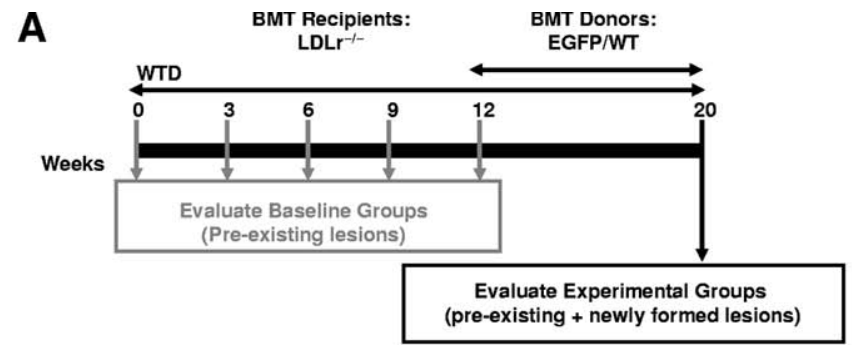

B

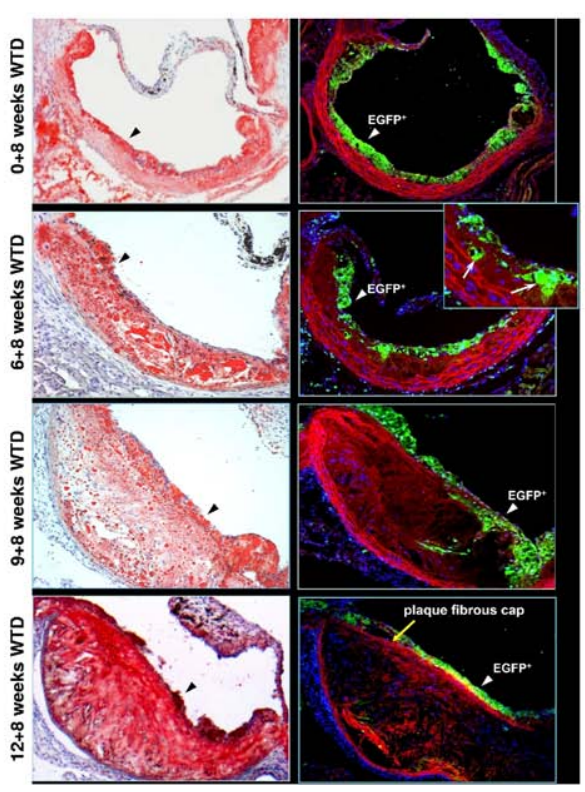

C
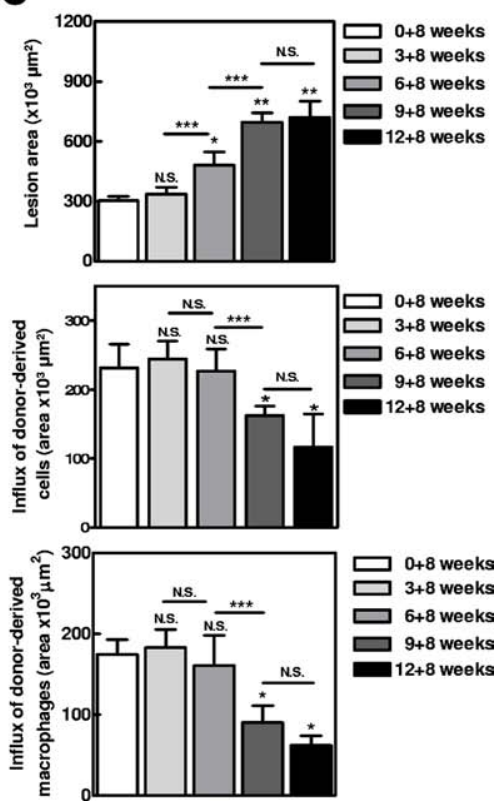

Figure 2. Generation of low-density lipoprotein receptor $(\mathrm{LDLr})^{-/-}$mice with pre-existing atherosclerotic lesions and overexpressing enhanced green fluorescent protein (EGFP) in macrophages. A: The experimental setup is demonstrated. Bone marrow from EGFP/wild type (WT) donors was transplanted into $\mathrm{LDLr}^{-/-}$mice that had been fed a Western-type diet (WTD) for $0,3,6,9$, and 12 weeks before bone marrow transplantation (BMT) (EGFP/WT $\rightarrow \mathrm{LDLr}^{-1-}$ ). The animals that underwent transplantation were sacrificed after $8,11,14,17$, and 20 weeks of WTD feeding in total (ie, 8 weeks of WTD feeding after BMT). Subsequently, the preexisting and newly formed atherosclerotic lesions were analyzed. B: Representative images for oil red O staining of the atherosclerotic lesions with freshly infiltrated donor-derived macrophages showing massive lipid accumulation (black arrows). Corresponding merged photomicrographs of EGFP (green), F4/80 (red) for macrophages, and 4,6-diamidino-2-phenylindole (blue) for nuclei in the aortic root lesions. Colocalization of macrophages with EGFP fluorescence is yellow-green (white arrows). A fibrous cap (yellow arrow) was clearly observed in pre-existing advanced fibroatheroma lesions, and almost no donor-derived macrophages were detected within these lesions (original magnification, $\times 200$ ). C: The mean lesion area was calculated from oil red $\mathrm{O} /$ hematoxylin-stained cross sections of the aortic root of EGFP-WT $\rightarrow$ $\mathrm{LDLr}^{-/-}$mice at the level of the tricuspid valves. The influx of donor-derived cells and donorderived macrophages was evaluated by quantifying the total $\mathrm{EGFP}^{+}$and $\mathrm{EGFP}^{+} \mathrm{F} 4 / 80^{+}$areas, respectively, in the aortic root of EGFP-WT $\rightarrow$ $\mathrm{LDLr}^{-1-}$ mice. Values represent the mean of 10 mice per group. ${ }^{*} P<0.05$ and ${ }^{* *} P<0.01$ versus mice that underwent transplantation and had no pre-existing lesions (ie, 0 - plus 8 -week WTD-fed mice). ${ }^{* * *} P<0.05$ versus the indicated group; NS, nonsignificant. 
Table 2. Serum Lipid Levels in the $\mathrm{LDLr}^{-/-}$Mice that Underwent Transplantation and Had Pre-Existing Atherosclerotic Lesions and Overexpressing EGFP in Macrophages*

\begin{tabular}{cccccc}
\hline Group & Time receiving a WTD, wk & $\mathrm{FC}, \mathrm{mg} / \mathrm{dL}^{\dagger}$ & $\mathrm{CE}, \mathrm{mg} / \mathrm{dL}^{\dagger}$ & $\mathrm{PL}, \mathrm{mg} / \mathrm{dL}^{\dagger}$ & $\mathrm{TG}^{\dagger} \mathrm{mg} / \mathrm{dL}^{\dagger}$ \\
\hline 1 & $0+8$ & $653 \pm 9$ & $870 \pm 30$ & $702 \pm 39$ & $326 \pm 58$ \\
2 & $3+8$ & $625 \pm 9$ & $913 \pm 14$ & $642 \pm 31$ & $270 \pm 49$ \\
3 & $6+8$ & $658 \pm 14$ & $1056 \pm 51$ & $529 \pm 31^{\ddagger}$ & $144 \pm 7^{\ddagger}$ \\
4 & $9+8$ & $671 \pm 17$ & $1264 \pm 74^{\S}$ & $614 \pm 33$ & $193 \pm 25$ \\
5 & $12+8$ & $680 \pm 18$ & $1303 \pm 68^{\S}$ & $620 \pm 30$ & $193 \pm 29$ \\
\hline
\end{tabular}

LDLr, low-density lipoprotein receptor; EGFP, enhanced green fluorescent protein; WTD, Western-type diet; FC, free cholesterol; CE, cholesterol ester; PL, phospholipid; TG, triglyceride.

${ }^{\star}$ For all groups, $n=10$. Before bone marrow transplantation (BMT), LDLr ${ }^{-1-}$ mice were fed a high-cholesterol WTD for 0, 3, 6, 9, and 12 weeks. Serum levels of FC, CE, PL, and TG were determined in the LDLr ${ }^{-1-}$ mice that underwent transplantation after $8,11,14,17$, and 20 weeks of WTD feeding in total (ie, 8 weeks of WTD feeding after BMT).

${ }^{\dagger}$ Data are given as mean \pm SEM.

${ }^{\ddagger} P<0.01$ versus LDLr ${ }^{-1-}$ mice that underwent transplantation and had no pre-existing lesions (group 1 )

$\S P<0.05$ versus $\mathrm{LDLr}^{-1-}$ mice that underwent transplantation and had no pre-existing lesions (group 1)

terol levels were still adequately high to induce further progression of the pre-existing atherosclerotic lesions. At sacrifice, EGFPNT $\rightarrow \mathrm{LDLr}^{-1-}$ mice without established lesions (ie, 0- plus 8-week WTD-fed mice) and the mice that underwent transplantation and had pre-existing initial lesions (ie, 3- plus 8-week or 6- plus 8-week WTD-fed mice) had similar serum levels of FC and CE. However, the mice that underwent transplantation and had pre-existing more advanced lesions (ie, 9- plus 8-week or 12- plus 8-week WTD-fed mice) displayed significantly $(P<0.05)$ higher levels of serum CE but not of FC (Table 2). The PL and TG levels were similar in all groups, except for the group that underwent transplantation, which had been fed a WTD for 6 weeks before BMT (ie, 6- plus 8-week WTD-fed mice), showing slightly lower PL and TG levels.

\section{Macrophage Infiltration into Pre-Existing Atherosclerotic Lesions with Different Degrees of Complexity}

Next, atherosclerotic lesion development was analyzed in the aortic root of EGFP/WT $\rightarrow \mathrm{LDLr}^{-1-}$ mice after 8,11 14,17 , and 20 weeks of WTD feeding in total (ie, 8 weeks of WTD feeding after BMT) (Figure 2, B and C). In the mice that underwent transplantation and had no preexisting lesions (ie, 0- plus 8-week WTD-fed mice), fatty streak lesions were formed $\left(304 \pm 20 \times 10^{3} \mu \mathrm{m}^{2}, n=\right.$ $10)$, mostly composed of macrophage-derived foam cells and no fibrous cap. In the mice that underwent transplantation and had been fed a WTD for 3 and 6 weeks before $\mathrm{BMT}$, pre-existing initial lesions had formed before transplantation $\left(31 \pm 7 \times 10^{3} \mu \mathrm{m}^{2}, n=4\right.$; and $190 \pm 43 \times 10^{3}$, $n=4$, respectively); these lesions progressed into larger lesions $\left(336 \pm 34 \times 10^{3} \mu \mathrm{m}^{2}, n=10\right.$; and $480 \pm 68 \times$ $10^{3} \mu \mathrm{m}^{2}, n=10$, respectively) at 8 weeks after BMT (ie, 3- plus 8-week and 6- plus 8-week WTD feeding, respectively). Moreover, in the mice that underwent transplantation and had been fed a WTD for 9 and 12 weeks before BMT, pre-existing more advanced lesions had formed $\left(505 \pm 51 \times 10^{3} \mu \mathrm{m}^{2}, n=4\right.$; and $600 \pm 11 \times 10^{3} \mu \mathrm{m}^{2}$, $n=4$, respectively), characterized by a low macrophage content, an increased collagen content, and fibrous cap formation. These pre-existing more advanced lesions increased only slightly in size $\left(696 \pm 46 \times 10^{3} \mu \mathrm{m}^{2}, n=10\right.$; and $720 \pm 82 \times 10^{3} \mu \mathrm{m}^{2}, n=10$, respectively) at 8 weeks after BMT (ie, 9- plus 8-week and 12- plus 8-week WTD feeding, respectively).

Immunohistochemical staining was performed to distinguish between the pre-existing lesions $\left(\mathrm{EGFP}^{-}\right)$and the newly formed lesions $\left(\mathrm{EGFP}^{+}\right)$. As shown, in EGFP/WT $\rightarrow \mathrm{LDLr}^{-1-}$ mice without pre-existing lesions (ie, 0-plus 8-week WTD-fed mice), the aortic lesions were exclusively composed of donor-derived EGFP-expressing cells, which were primarily $\mathrm{F} 4 / 80^{+}$macrophages (Figure $2 \mathrm{~B}$ ). In contrast, in EGFP/WT $\rightarrow \mathrm{LDLr}^{-1-}$ mice with pre-existing initial lesions (ie, 6- plus 8-week WTD-fed mice), the fatty streak lesions had grown partly by forming a new layer of $\mathrm{EGFP}^{+} \mathrm{F} 4 / 80^{+}$donor-derived macrophages above the pre-existing EGFP- lesions. Furthermore, a few donor-derived macrophages had infiltrated inside the pre-existing initial lesions (Figure 2B). Interestingly, in EGFP/WT $\rightarrow \mathrm{LDLr}^{-1-}$ mice with pre-existing more advanced lesions (ie, 9- plus 8-week WTD-fed mice), an influx of donor-derived cells, including $\mathrm{F} 4 / 80^{+}$ macrophages, into the arterial wall was largely blocked because only a thin layer of newly infiltrated cells had accumulated on top of the fibrous cap of the established complex lesions (Figure 2B). Furthermore, almost no donor-derived cells were detected within the pre-existing advanced fibroatheroma lesions (ie, 12- plus 8-week WTD-fed mice) (Figure 2B). By quantifying the $\mathrm{EGFP}^{+} \mathrm{F} 4 / 80^{+}$areas, we did not find a significant difference in the influx of donor-derived macrophages into the arterial wall of the mice that underwent transplantation and had no pre-existing lesions (ie, 0- plus 8-week WTD-fed mice) compared with those with preexisting initial lesions (ie, 3- plus 8-week or 6- plus 8-week WTD-fed mice) (Figure 2C). In total, 78\% \pm $22 \%$ of the infiltrated $\mathrm{EGFP}^{+}$cells were $\mathrm{F} 4 / 80^{+}$macrophages. The remaining percentage will include $\mathrm{F} 4 / 80^{-}$ macrophages, dendritic cells, T cells, neutrophils, and mast cells. Interestingly, a significantly $(P<0.05)$ lower influx of donor-derived macrophages into the arterial wall was observed in the mice that underwent transplantation and had pre-existing more advanced lesions (ie, 9- plus 8-week or 12-plus 8-week WTD-fed mice). Under these conditions, the percentage of $\mathrm{EGFP}^{+}$cells that also expressed F4/80 had decreased 

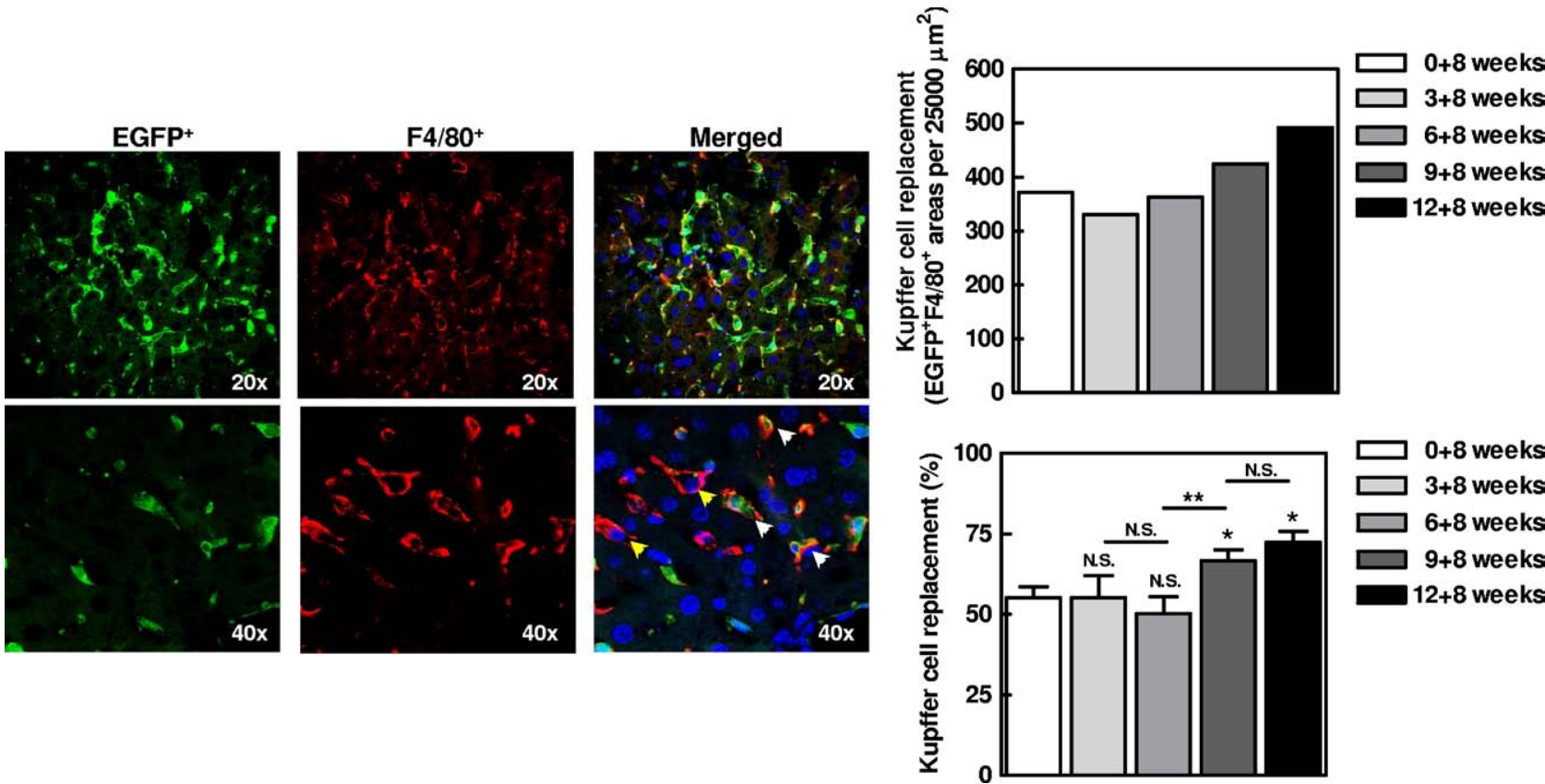

Figure 3. Replacement of Kupffer cells in the liver of low-density lipoprotein receptor (LDLr) atherosclerotic lesions with different degrees of complexity. Representative merged photomicrographs of enhanced green fluorescent protein (EGFP) (green), F4/80 (red) for Kupffer cells (hepatic resident macrophages), and 4,6-diamidino-2-phenylindole (DAPI) (blue) for nuclei in the liver of EGFP-wild type (WT) $\rightarrow$ LDLr $^{-/-}$mice. Colocalization of Kupffer cells with EGFP fluorescence (thus, of donor origin) is yellow-green (white arrows). Kupffer cells without EGFP fluorescence (thus, of recipient origin) are red (yellow arrows) (original magnification, $\times 400$ ). The density of $\mathrm{F} / 80^{+}$and $\mathrm{EGFP}^{+} \mathrm{F} / 80^{+}$cells was calculated as the number of stained cells per $25,000 \mu \mathrm{m}^{2}$ in the liver of EGFP-WT $\rightarrow$ LDLr $^{-1-}$ mice. Regions of interest were selected blindly using DAPI staining as a reference. Values represent the average from at least five adjacent sections. We further determined the ratio $\mathrm{EGFP}^{+} \mathrm{F} 4 / 80^{+} \mathrm{cells} / \mathrm{F} / 80^{+}$cells, indicative for the replacement of Kupffer cells. Values represent the mean of 10 mice per group. ${ }^{*} P<0.05$ versus mice that underwent transplantation and had no pre-existing lesions (ie, 0 - plus 8 -week WTD-fed mice). ${ }^{* *} P<0.05$ versus the indicated group; NS, nonsignificant.

to $55 \% \pm 7 \%$ and $52 \% \pm 24 \%$ of the total amount of $\mathrm{EGFP}^{+}$cells, respectively. These results suggest that the dynamics of cellular infiltration and more specific macrophage infiltration into the arterial wall are largely impaired in more advanced atherosclerotic lesions.

\section{Replacement of Liver, Spleen, and Lymph Node Resident Macrophages in LDLr ${ }^{-1-}$ Mice That Underwent Transplantation}

The liver contains the most abundant macrophage population in the body. At 8 weeks after BMT, much of the $\mathrm{F} 4 / 80^{+}$resident macrophages in the liver Kupffer cells expressed EGFP and, thus, were of donor origin (Figure 3). Calculation of the ratio of $\mathrm{EGFP}^{+} \mathrm{F} 4 / 80^{+}$cells/F4/ $80^{+}$cells, indicative for the replacement of Kupffer cells, showed that greater than $50 \%$ of the Kupffer cells were replaced. Interestingly, significantly $(P<0.05)$ higher replacement of Kupffer cells was observed in the liver of EGFP-WT $\rightarrow \mathrm{LDLr}^{-1-}$ mice with pre-existing more advanced lesions (ie, 9- plus 8-week or 12- plus 8-week WTDfed mice) than those without pre-existing lesions (ie, 0- plus 8-week WTD-fed mice) or with pre-existing initial lesions (ie, 3- plus 8-week or 6- plus 8-week WTD-fed mice).

In addition, higher replacement of resident macrophages in the spleen and lymph nodes was found in the mice that underwent transplantation and had pre-existing more advanced lesions than in those without pre-existing lesions or with pre-existing initial lesions, as determined by FACS analysis (see Supplemental Figure S2 at $h$ ttp://ajp.amjpathol. org). Thus, the observed impaired infiltration of macrophages into more advanced atherosclerotic lesions is not a consequence of generally impaired macrophage infiltration into tissues. Actually, macrophage infiltration into other organs was increased, probably as a result of enhanced inflammatory status in those animals that underwent transplantation and had pre-existing more advanced lesions.

\section{Effect of Macrophage ABCA1 Expression on the} Development of Pre-Existing Atherosclerotic Lesions in $L D L r^{-1-}$ Mice

Previously, a protective role was demonstrated for macrophage ABCA1 in atherosclerosis development in $\mathrm{LDLr}^{-1-}$ mice without pre-existing lesions, ${ }^{9,10}$ and disruption of $A B C A 1$ in hematopoietic cells resulted in enhanced recruitment of macrophages into multiple tissues (eg, liver and spleen). ${ }^{9}$ To reveal the effect of ABCA1 expression on the infiltration of macrophages into preexisting atherosclerotic lesions, bone marrow from EGFP/ ABCA1 KO, EGFP/hABCA1, and EGFP/WT mice was transplanted into $\mathrm{LDLr}^{-1-}$ mice that had been fed a WTD for 6 and 9 weeks before BMT (EGFP/ABCA1 KO $\rightarrow$ $\mathrm{LDLr}^{-1-}, \mathrm{EGFP} / \mathrm{hABCA} 1 \rightarrow \mathrm{LDLr}^{-\prime-}$, and EGFP/WT $\rightarrow$ $\mathrm{LDLr}^{-1-}$, respectively) and subsequently challenged for another 15 and 12 weeks with a WTD, respectively (Figure 4A).

Successful reconstitution of recipients with donor-derived cells was established at 12 or 15 weeks after BMT. 
A BMT Recipients:

BMT Donors:

EGFP/ABCA1 KO, EGFP/hABCA1, and EGFP/WT

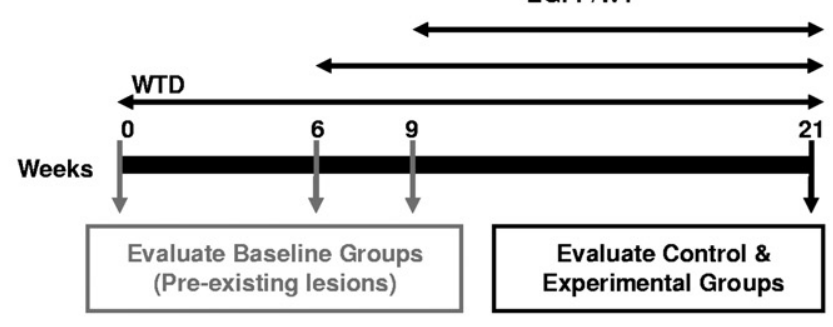

B

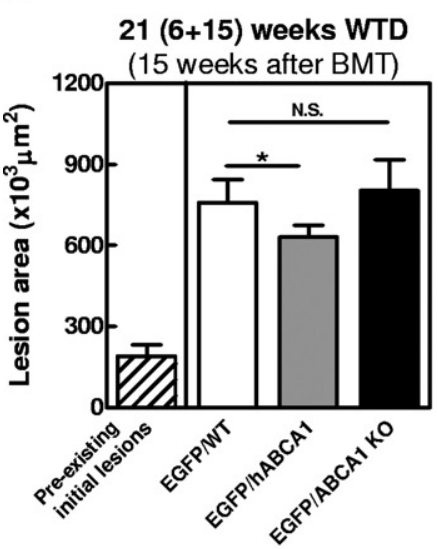

$21(9+12)$ weeks WTD

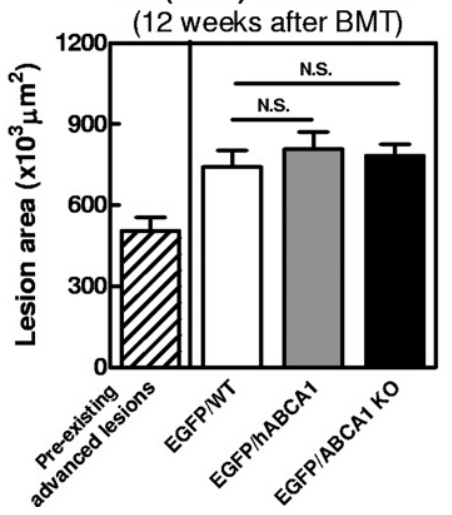

C

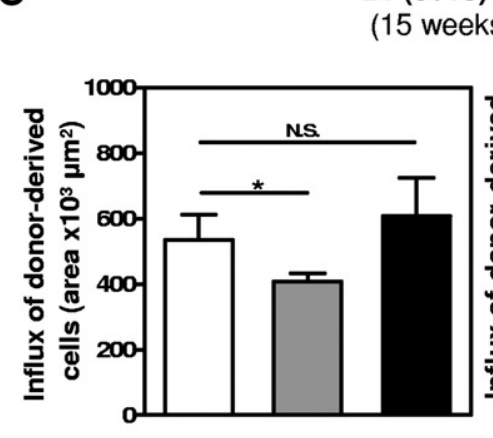

Figure 4. The effect of macrophage ABCA1 expression on the development of pre-existing atherosclerotic lesions in $\mathrm{LDLr}^{-1-}$ mice. A: The experimental setup is demonstrated. Bone marrow from EGFP/ABCA1 KO, EGFP/hABCA1, and EGFP/WT mice was transplanted into $\mathrm{LDLr}^{-/-}$mice that had been fed a WTD for 6 and 9 weeks before BMT (EGFP/ABCA1 KO $\rightarrow \mathrm{LDLr}^{-/-}$, EGFP/ hABCA1 $\rightarrow \mathrm{LDLr}^{-/}$, and EGFP/WT $\rightarrow \mathrm{LDLr}^{-1-}$ ) and subsequently challenged for another 15 and 12 weeks with a WTD, respectively. B: The mean lesion area was calculated from oil red $\mathrm{O} /$ hematoxylin-stained cross sections of the aortic root of mice that underwent transplantation and had pre-existing initial lesions or pre-existing more advanced lesions. ${ }^{*} P=0.05 \mathbf{C}$ : The influx of donor-derived cells and macrophages was evaluated by quantifying the total $\mathrm{EGFP}^{+}$and $\mathrm{EGFP}^{+} \mathrm{F} 4 / 80^{+}$areas in the aortic root of animals that underwent transplantation. Values represent the mean of 8 to 12 mice per group. ${ }^{*} P<0.05$ versus EGFP/WT mice that underwent transplantation. NS indicates nonsignificant.

$21(6+15)$ weeks WTD
$(15$ weeks after BMT)

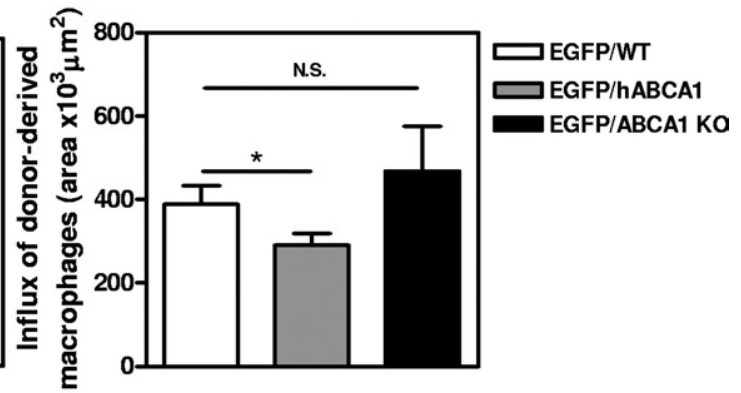

$21(9+12)$ weeks WTD

(12 weeks after BMT)
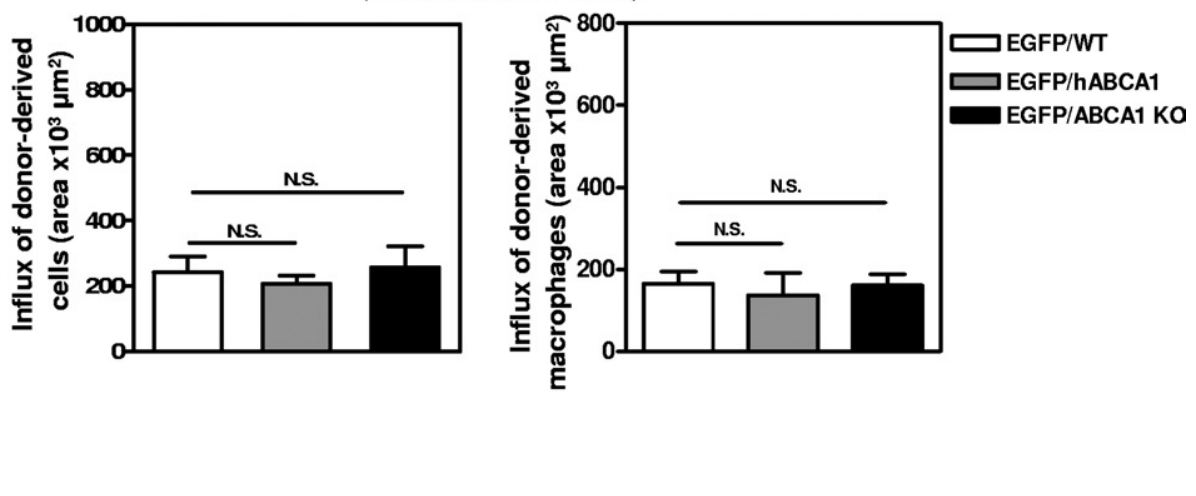

Genomic DNA isolated from the EGFP/ABCA1 KO $\rightarrow$ $\mathrm{LDLr}^{-/-}, \mathrm{EGFP} / \mathrm{hABCA} 1 \rightarrow \mathrm{LDLr}^{-/-}$, and EGFP/WT $\rightarrow$ $\mathrm{LDLr}^{-/-}$mice contained the ABCA1-null mutant, the human $A B C A 1$, and only the murine $A B C A 1$ gene, respectively (see Supplemental Figure S3 at $h$ ttp://ajp.amjpathol.org). Independently of the presence of pre-existing lesions, macrophage ABCA1 expression did not significantly affect serum high-density lipoprotein cholesterol levels (data not shown), which is in line with previous findings. 9,10

Atherosclerotic lesion development was analyzed in the aortic root of EGFP/WT $\rightarrow \mathrm{LDLr}^{-1-}$, EGFP/hABCA1 $\rightarrow \mathrm{LDLr}^{-1-}$, and EGFP/ABCA1 KO $\rightarrow \mathrm{LDLr}^{-1-}$ mice after 21 weeks of WTD feeding in total (ie, 15 and 12 weeks of WTD feeding after BMT). As shown in Figure $4 \mathrm{~B}$, macrophage ABCA1 deficiency did not significantly affect the development of pre-existing initial lesions (lesion areas, $803 \pm 113 \times 10^{3} \mu \mathrm{m}^{2}, n=12$; and
$758 \pm 86 \times 10^{3} \mu \mathrm{m}^{2}, n=12$ [for EGFP/ABCA1 KO $\rightarrow$ $\mathrm{LDLr}^{-1-}$ mice and EGFP/WT $\rightarrow \mathrm{LDLr}^{-1-}$ controls, respectively]; $P=0.13)$, whereas macrophage ABCA1 overexpression moderately $(P=0.05)$ inhibited the progression of pre-existing initial lesions (lesion area, $631 \pm 42 \times 10^{3} \mu \mathrm{m}^{2}, n=8$, for EGFP/hABCA1 $\rightarrow$ $\mathrm{LDLr}^{-1-}$ mice). On pre-existing advanced lesions, no effect of deletion or overexpression of macrophage ABCA 1 was observed (lesion areas, $783 \pm 42 \times 10^{3}$ $\mu \mathrm{m}^{2}[n=12], 807 \pm 63 \times 10^{3} \mu \mathrm{m}^{2}[n=8]$, and $742 \pm$ $60 \times 10^{3} \mu \mathrm{m}^{2}[n=10]$ for EGFP/ABCA1 KO $\rightarrow \mathrm{LDLr}^{-1-}$ mice, EGFP/hABCA1 $\rightarrow \mathrm{LDLr}^{-/-}$mice, and EGFP/WT $\rightarrow \mathrm{LDLr}^{-1-}$ controls, respectively).

By quantifying the $\mathrm{EGFP}^{+} \mathrm{F} 4 / 80^{+}$areas in the lesions, we observed that influx of ABCA1-deficient macrophages into the pre-existing initial lesions was not significantly changed $\left(\mathrm{EGFP}^{+} \mathrm{F} 4 / 80^{+}\right.$areas, $468 \pm 107 \times 10^{3} \mu \mathrm{m}^{2}$ 
$[n=12]$ and $389 \pm 44 \times 10^{3} \mu \mathrm{m}^{2}[n=12]$ for EGFP/ $\mathrm{ABCA} 1 \mathrm{KO} \rightarrow \mathrm{LDLr}^{-1-}$ mice and EGFP/WT $\rightarrow \mathrm{LDLr}^{-/-}$ controls, respectively), whereas the influx of ABCA1overexpressing macrophages into the pre-existing initial lesions was significantly $(P<0.05)$ impaired $\left(\mathrm{EGFP}^{+} \mathrm{F} 4 /\right.$ $80^{+}$area, $291 \pm 28 \times 10^{3} \mu \mathrm{m}^{2}[n=8]$ for EGFP/hABCA1 $\rightarrow \mathrm{LDLr}^{-1-}$ mice) (Figure $4 \mathrm{C}$ ). Independent of the type of donor bone marrow used, EGFP ${ }^{+} \mathrm{F} 4 / 80^{+}$cells composed approximately $75 \%$ of the total amount of $\mathrm{EGFP}^{+}$infiltrated cells. As expected, the infiltration of bone marrowderived macrophages into the pre-existing more advanced lesions was substantially lower than that into the pre-existing initial lesions. More important, the influx of donor-derived macrophages into the established more advanced lesions was not affected by macrophage ABCA1 expression $\left(\right.$ EGFP $^{+} \mathrm{F} 4 / 80^{+}$areas, $161 \pm 28 \times$ $10^{3} \mu \mathrm{m}^{2}[n=12], 165 \pm 29 \times 10^{3} \mu \mathrm{m}^{2}[n=8]$, and $137 \pm 54 \times 10^{3} \mu \mathrm{m}^{2}[n=10]$ for EGFP/ABCA1 $\mathrm{KO} \rightarrow$ $\mathrm{LDLr}^{-/-}$mice, EGFP/hABCA1 $\rightarrow \mathrm{LDLr}^{-1-}$ mice, and EGFP/WT $\rightarrow \mathrm{LDLr}^{-1-}$ controls, respectively). Moreover, the total amount of EGFP ${ }^{+}$cells that had infiltrated the lesions was not affected.

\section{Discussion}

In this study, BMT was used to investigate the dynamics of macrophage infiltration into the arterial wall during the pathogenesis of atherosclerosis. By tracking donor-derived macrophages (EGFP ${ }^{+} \mathrm{F} 4 / 80^{+}$), we demonstrate the following: (1) the growth of pre-existing early lesions is mainly caused by continued infiltration of macrophages into the arterial wall; (2) macrophage infiltration into preexisting more advanced lesions is largely impaired, most likely because of the formation of a fibrous cap; and (3) macrophage ABCA1 expression affects the infiltration of macrophage into pre-existing early lesions but not into more advanced lesions.

After transplantation, pre-existing fatty streak lesions largely increased in size, which was mainly caused by continued infiltration of donor-derived macrophages into the arterial wall. Moreover, we found that influx of

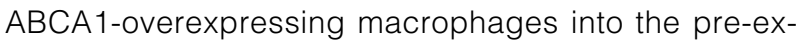
isting fatty streak lesions was significantly impaired. The ABCA1-mediated cholesterol efflux is a key factor in preventing the accumulation of macrophage foam cells in the arterial wall. In addition, ABCA1 modulates cholesterol content both on the cell surface and within intracellular compartments, thereby affecting macrophage function by influencing cellular inflammatory cytokine secretion. ${ }^{14-18}$ However, not all of the anti-inflammatory properties of $\mathrm{ABCA} 1$ are a consequence of its lipid transport activity. ATP-binding cassette transporter 1 interacts with its acceptor (ie, apolipoprotein A-I mimetic peptides) and activates signaling molecules (ie, Janus kinase 2). ${ }^{19-22}$ Thus, macrophage $A B C A 1$ exerts multiple antiatherogenic functions that may explain the inhibitory effect of ABCA 1 overexpression on the infiltration of macrophages into pre-existing fatty streak lesions, resulting in reduced lesion size. These findings suggest that preventing macrophage recruitment into early stages of lesion development provides a promising therapeutic strategy to reduce atherosclerosis burden and that increasing macrophage $A B C A 1$ expression may be one avenue of doing so. Surprisingly, no significant effect of ABCA1 deficiency was observed on macrophage recruitment into pre-existing early lesions. Bone marrow transplantation has affected atherosclerotic lesion development. ${ }^{23}$ Therefore, the lack of an effect of macrophage ABCA1 $\mathrm{KO}$ on lesion progression and macrophage infiltration may be because of BMT-dependent effects on lesion progression.

Pre-existing more advanced lesions increased only slightly in size after transplantation, and the infiltration of donor-derived macrophages into these lesions was largely impaired. In mice with pre-existing advanced lesions, we observed a significantly higher replacement of tissue macrophages compared with mice without pre-existing lesions or with pre-existing early lesions. Wouters et $\mathrm{al}^{24}$ demonstrated that dietary cholesterol can provoke hepatic inflammation in hyperlipidemic mice, possibly because of direct activation of Kupffer cells on scavenging remnant lipoproteins. More recently, the effect of dietary cholesterol on hepatic inflammation was further elucidated by the same group; it was suggested that intrahepatic cholesterol influences progression, inhibition, and reversal of nonalcoholic steatohepatitis in hyperlipidemic mice. ${ }^{25}$ In this study, we found that $\mathrm{LDLr}^{-/-}$mice with pre-existing more advanced lesions displayed higher levels of intrahepatic cholesterol, which may trigger an inflammatory response in the liver of these animals. Our findings also indicate that the impaired influx of macrophages into atherosclerotic lesions of mice with preexisting more advanced lesions is not the consequence of a reduced capacity of macrophages to infiltrate into tissues under these conditions. Lesion progression coincides with the formation of a fibrous cap, which is essential for the stability of the plaque. It is most likely that the formation of fibrous caps in preexisting advanced lesions directly prevents further influx of macrophages into the arterial wall. More important, our results indicate that therapeutic modulation of macrophage infiltration would not be expected to have beneficial effects on more advanced lesions. We show that neither disruption nor up-regulation of macrophage ABCA1 expression significantly influences the development of atherosclerotic lesions in mice with pre-existing more advanced lesions.

In conclusion, we established a new model for studying the dynamic changes in lesion development using BMT. The BMT procedure and, in particular, the irradiation required to eliminate the endogenous bone marrow cells might affect the atherogenic process. However, using this model, for the first time, we were able to show that the dynamics of macrophage infiltration into the arterial wall largely differ during atherosclerotic lesion development. This may affect the efficiency of pharmaceutical interventions aimed at targeting macrophage infiltration into the arterial wall. 


\section{References}

1. Bennett MR: The atherosclerotic plaque was not built in a day: the dynamic nature of plaque progression and instability. Heart Metab 2007, 36:5-7

2. Consensus conference: lowering blood cholesterol to prevent heart disease. JAMA. 1985, 253:2080-2086

3. Smith JD, Trogan E, Ginsberg M, Grigaux C, Tian J, Miyata M: Decreased atherosclerosis in mice deficient in both macrophage colony-stimulating factor (op) and apolipoprotein E. Proc Natl Acad Sci U S A 1995, 92:8264-8268

4. McKnight AJ, Gordon S: Membrane molecules as differentiation antigens of murine macrophages. Adv Immunol 1998, 68:271-314

5. Vu TH, Werb Z: Matrix metalloproteinases: effectors of development and normal physiology. Genes Dev 2000, 14:2123-2133

6. Galis ZS, Sukhova GK, Lark MW, Libby P: Increased expression of matrix metalloproteinases and matrix degrading activity in vulnerable regions of human atherosclerotic plaques. J Clin Invest 1994, 94: 2493-2503

7. Oram JF: HDL apolipoproteins and ABCA1: partners in the removal of excess cellular cholesterol. Arterioscler Thromb Vasc Biol 2003, 23: 720-727

8. Francis GA, Knopp RH, Oram JF: Defective removal of cellular cholesterol and phospholipids by apolipoprotein A-I in Tangier disease. J Clin Invest 1995, 96:78-87

9. Van Eck M, Bos IS, Kaminski WE: Leukocyte ABCA1 controls susceptibility to atherosclerosis and macrophage recruitment into tissues. Proc Natl Acad Sci U S A 2002, 99:6298-6303

10. Van Eck M, Singaraja RR, Ye D, Hildebrand RB, James ER, Hayden MR, Van Berkel TJ: Macrophage ATP-binding cassette transporter A1 overexpression inhibits atherosclerotic lesion progression in low-density lipoprotein receptor knockout mice. Arterioscler Thromb Vasc Biol 2006, 26:929-934

11. Schaefer BC, Schaefer ML, Kappler JW, Marrack P, Kedl RM: Observation of antigen-dependent CD8+ T-cell/dendritic cell interactions in vivo. Cell Immunol 2001, 214:110-122

12. Stary HC, Chandler AB, Glagov S, Guyton Jr, Insull W Jr, Rosenfeld ME, Schaffer SA, Schwartz CJ, Wagner WD, Wissler RW: A definition of initial, fatty streak, and intermediate lesions of atherosclerosis: a report from the Committee on Vascular Lesions of the Council on Arteriosclerosis, American Heart Association. Circulation 1994, 89: 2462-2478

13. Bligh EG, Dyer WJ: A rapid method of total lipid extraction and purification. Can J Biochem Physiol 1959, 37:911-917

14. Zhu X, Lee JY, Timmins JM, Brown JM, Boudyguina E, Mulya A Gebre AK, Willingham MC, Hiltbold EM, Mishra N, Maeda N, Parks JS: Increased cellular free cholesterol in macrophage-specific Abca1 knock-out mice enhances pro-inflammatory response of macrophages. J Biol Chem 2008, 283:22930-22941

15. Koseki M, Hirano K, Masuda D, Ikegami C, Tanaka M, Ota A, Sandoval JC, Nakagawa-Toyama Y, Sato SB, Kobayashi T, Shimada Y, Ohno-Iwashita Y, Matsuura F, Shimomura I, Yamashita S: Increased lipid rafts and accelerated lipopolysaccharide-induced tumor necrosis factor-alpha secretion in Abca1-deficient macrophages. J Lipid Res 2007, 48:299-306

16. Sun $Y$, Ishibashi M, Seimon $T$, Lee M, Sharma SM, Fitzgerald KA Samokhin AO, Wang Y, Sayers S, Aikawa M, Jerome WG, Ostrowsk MC, Bromme D, Libby P, Tabas IA, Welch CL, Tall AR: Free cholesterol accumulation in macrophage membranes activates Toll-like receptors and p38 mitogen-activated protein kinase and induces cathepsin K. Circ Res 2009, 104:455-465

17. Lajoie P, Goetz JG, Dennis JW, Nabi IR: Lattices, rafts, and scaffolds: domain regulation of receptor signaling at the plasma membrane. J Cell Biol 2009, 185:381-385

18. Strauss JF 3rd, Liu P, Christenson LK, Watari H: Sterols and intracellular vesicular trafficking: lessons from the study of NPC1. Steroids 2002, 67:947-951

19. Tang C, Vaughan AM, Anantharamaiah GM, Oram JF: Janus kinase 2 modulates the lipid-removing but not protein-stabilizing interactions of amphipathic helices with ABCA1. J Lipid Res 2006, 47:107-114

20. Tang C, Vaughan AM, Oram JF: Janus kinase 2 modulates the apolipoprotein interactions with $\mathrm{ABCA} 1$ required for removing cellular cholesterol. J Biol Chem 2004, 279:7622-7628

21. Aaronson DS, Horvath CM: A road map for those who don't know JAK-STAT. Science 2002, 296:1653-1655

22. Tang C, Liu Y, Kessler PS, Vaughan AM, Oram JF: The macrophage cholesterol exporter ABCA1 functions as an anti-inflammatory receptor. J Biol Chem 2009, 284:32336-32343

23. Schiller NK, Kubo N, Boisvert WA, Curtiss LK: Effect of gammairradiation and bone marrow transplantation on atherosclerosis in LDL receptor-deficient mice. Arterioscler Thromb Vasc Biol 2001, 21 $1674-1680$

24. Wouters K, van Gorp PJ, Bieghs V, Gijbels MJ, Duimel H, Lütjohann D, Kerksiek A, van Kruchten R, Maeda N, Staels B, van Bilsen M, Shiri-Sverdlov $\mathrm{R}$, Hofker $\mathrm{MH}$ : Dietary cholesterol, rather than liver steatosis, leads to hepatic inflammation in hyperlipidemic mouse models of nonalcoholic steatohepatitis. Hepatology 2008, 48:474486

25. Wouters K, van Bilsen M, van Gorp PJ, Bieghs V, Lütjohann D, Kerksiek A, Staels B, Hofker MH, Shiri-Sverdlov R: Intrahepatic cholesterol influences progression, inhibition and reversal of non-alcoholic steatohepatitis in hyperlipidemic mice. FEBS Lett 2010, 584 1001-1005 